\title{
Future role of CHWs in improving maternal and child health
}

\author{
Fareeha Siddui, Zahra Padhani and Jai K Das* \\ Division of Women and Child Health, Aga Khan University, Karachi, Pakistan
}

Community health workers (CHWs) are paraprofessionals or lay health workers, recruited from and based within the communities that they serve. They are local residents, who undertake short trainings on healthcare and acquire relevant knowledge and skills to provide culturally specific health services. They are frontline workers responsible for providing preventive, promotive and basic curative services at community level [1-5].

Following the Alma Ata Declaration of 1978, a paradigm shift in the discourse of public health was observed with the notion of preventive care being viewed as equally significant to curative care. Signatories of the Alma Ata, to display their commitment to the proposed policy of Primary Health Care (PHC), launched programs to involve lay people within the community to deliver healthcare. The consequence was the proliferation of the CHW programs, with many governments and international organizations seeking to pursue and support it. However during the 1990's, a reversal trend was noted with CHW programs gradually declining. This could be attributed to certain policy shifts which focused upon the cost-effectiveness, as well as, the lack of concrete evidence supporting the contribution and efficacy of the programs [3].

More recently, against the backdrop of increased disease burden, there has been a re-awakened interest in $\mathrm{CHW}$ initiatives, especially in the developing world. The purpose of revitalizing this initiative is to address the shortage of health professionals by involving local community members in the process of healthcare delivery. Moreover, with the growth of empowerment literature, there is a greater inclination to view CHW personnel as 'agents of change.' This is primarily because of their strong engagement with the community and their role in activities of community mobilization and development $[3,6]$.

A primary role CHWs play is to provide maternal and child health services. This includes care from antenatal to postnatal stage. With ninety-nine percent of maternal and child mortality occurring in low-to-middle income countries; CHWs are found to be effective in resource-poor settings to reduce maternal, neonatal and child mortality [7]. Within the domain of $\mathrm{MCH}$, in terms of preventive measures, $\mathrm{CHWs}$ are responsible for a multitude of interventions including health promotion and disease prevention, breastfeeding intervention, family planning and newborn care. These services have aided in reducing levels of maternal and child mortality, while simultaneously also decreased the incidence of diseases, such as, Tuberculosis and Malaria. However, regardless of these improvements, the overall progress has been largely restricted.

The question that now arises is regarding the future role of CHWs in improving maternal and child health outcomes? - It seems evident that in the immediate future CHWs will continue to play an integral part in influencing maternal and child health outcomes. Reason being,
CHW's are recruited from within the community, and are trained to provide culturally appropriate health services [2]. This makes CHWs more likely to be well-accepted and appreciated by the community, especially women.

These frontline workers continue to serve as a vital liaison between the community and the formal healthcare system. By providing healthcare to the doorsteps of individuals, access to health becomes more equitable [8]. CHWs position as a healthcare provider remains significant, especially in the developing world, because of the gap that exists due to the mismatch between increased disease burden and inadequate skilled health practitioners. Being a local member of the community, CHWs have an in-depth understanding of practices within the community, allowing them to better understand barriers to healthcare [3]. This is important in terms of $\mathrm{MCH}$, because to improve maternal and child health outcomes it becomes pivotal for healthcare professionals to understand grass-root realities. In many developing countries, gendered inequities and its interaction with politics, class and caste plays an essential role in impacting women's access to healthcare. Consequently, this influences women's reproductive health. CHWs role as facilitators and community partners makes them better equipped to recognize and respond to these barriers, as they often share concerns and have greater sensitivity with their catchment populace $[3,8,9]$.

Briefly put, it is quite probable that in the immediate future, the $\mathrm{CHW}$ cadre due to its integral link with the community will continue to serve as a tool for improving $\mathrm{MCH}$ outcomes. However, the point of concern is regarding the degree of its effectiveness. The success of CHW programs depends greatly upon the political and socioeconomic context of the respective country. The greater the political will to support and bolster the program, the higher the likelihood of its success. Conducive political atmosphere contributes to program sustainability, especially when $\mathrm{CHW}$ programs are viewed as a complementary arm of the overall formal healthcare system. Additionally, cultural factors along with community support and acceptance considerably determine the success of CHW programs. Unless, a strong connection exists between the $\mathrm{CHW}$ personnel and the catchment populace, the initiative would not be successful [3].

The future role of CHWs in improving $\mathrm{MCH}$ outcomes rests upon certain do's and don'ts. The former requires supportive supervision of

${ }^{*}$ Correspondence to: Jai K Das, Division of Women and Child Health, Aga Khan University, Stadium Road Karachi, Pakistan, E-mail: jai.das@aku.edu

Received: December 02, 2019; Accepted: December 15, 2019; Published: December 17, 2019 
CHWs, continuous education and skill development, logistical support, and community's sense of ownership of the CHW program. The latter includes avoidance of hasty planning, poor communication, distorted selection criteria, lack of support from the health system and a sense of exploitation and isolation among the workers due to issues such as lack of recognition and problems relating to adequate remuneration. Therefore, if these do's and don'ts are maintained adequately, then the CHW cadre will serve as an effective tool for improving maternal and child health in the future [4].

\section{References}

1. Malcarney MB, Pittman P, Quigley L, Horton K, Seiler N (2017) The changing roles of community health workers. Health Serv Res 52: 360-382. [Crossref]

2. Olaniran A, Madaj B, Bar-Zev S, van den Broek N (2019) The roles of community health workers who provide maternal and newborn health services: case studies from Africa and Asia. BMJ Glob Health 4: e001388. [Crossref]

3. Rifkin SB (2017) Community Health Workers International Encyclopedia of Public Health ( $\left.2^{\text {nd }} E d n\right) .93-102$.
4. Strodel RJ, Perry HB (2019) The National Village Health Guide Scheme in India: lessons four decades later for community health worker programs today and tomorrow. Human resources for Health 17: 1-8. [Crossref]

5. Woldie M, Feyissa GT, Admasu B, Hassen K, Mitchell K, et al. (2018) Community health volunteers could help improve access to and use of essential health services by communities in LMICs: an umbrella review. Health Policy Plan 33: 1128-1143. [Crossref]

6. Bigirwa P (2009) Effectiveness of community health workers (CHWS) in the provision of basic preventive and curative maternal, newborn and child health (MNCH) interventions: A systematic review.

7. Gilmore B, McAuliffe E (2013) Effectiveness of community health workers delivering preventive interventions for maternal and child health in low-and middle-income countries: a systematic review. BMC Public Health 13: 847. [Crossref]

8. Wilford A, Phakathi S, Haskins L, Jama N, Mntambo N, et al. (2018) Exploring the care provided to mothers and children by community health workers in South Africa: missed opportunities to provide comprehensive care. BMC Public Health 18: 171. [Crossref]

9. Mumtaz Z, Salway S, Shanner L, Bhatti A, Laing L (2011) Maternal deaths in Pakistan: intersection of gender, caste, and social exclusion. BMC Int Health Hum Rights 11 S4. [Crossref]

Copyright: (C2019 Padhani FSZ. This is an open-access article distributed under the terms of the Creative Commons Attribution License, which permits unrestricted use, distribution, and reproduction in any medium, provided the original author and source are credited. 